\title{
Numerical Simulation: Effects of Gas Flow and Rf Current Direction on Plasma Uniformity in an ICP Dry Etcher
}

\author{
Junghoon Joo* \\ Department of Materials Science and Engineering, Kunsan National University, Gunsan, Jeonbuk 573-701, Korea
}

Received October 22, 2017; revised November 23, 2017; accepted November 24, 2017

\begin{abstract}
Effects of gas injection scheme and rf driving current configuration in a dual turn inductively coupled plasma (ICP) system were analyzed by 3D numerical simulation using CFD-ACE+. Injected gases from a tunable gas nozzle system (TGN) having 12 horizontal and 12 vertical nozzles showed different paths to the pumping surface. The maximum velocity from the nozzle reached Mach 2.2 with 2.2 $\mathrm{Pa}$ of Ar. More than half of the injected gases from the right side of the TGN were found to go to the pump without touching the wafer surface by massless particle tracing method. Gases from the vertical nozzle with 45 degree slanted angle soared up to the hottest region beneath the ceramic lid between the inner and the outer rf turn of the antenna. Under reversed driving current configuration, the highest $\mathrm{rf}$ power absorption region were separated into the two inner islands and the four peaked donut region.
\end{abstract}

Keywords: Plasma, Etching, Gas flow, Rf current, Uniformity, ICP

\section{Introduction}

High density plasma sources are widely used in semiconductor and other materials processing systems [110] Dry etchers are adopting fairly complicated rf delivery system [11]. A dual coil rf antenna configuration is one of them. The outer and inner turn can drive current either in same or opposite direction. Each one has much different plasma density profiles. Gas injection has a deep impact on processing results, e.g. etch rate profile via ion and radical generation as well as diffusion of reactive species. AMAT's DPS (decoupled plasma source) has been used both in poly-Si and metal etching steps. It has a dual turn $\mathrm{rf}$ antenna and a tunable gas nozzle (TGN). A numerical model was developed by using ESI's multiphysics simulation software, $\mathrm{CFD}-\mathrm{ACE}+$. Two main factors were investigated by using this numerical model: gas injection conditions and $\mathrm{rf}$ driving current direction (same and opposite).

\section{Experiment and Simulation Setup}

The overall configuration of the geometry model is shown in Fig. 1. CFD-ACE+ is using a fluid model: drift diffusion equation and quasi-neutral approximation in 3D models. Grid shapes can be either hexahedral or tetrahedral. Hexa cells give more stable convergence in any case. Otherwise specified, hexa cells were used in this

*Corresponding author

E-mail: jhjoo@kunsan.ac.kr

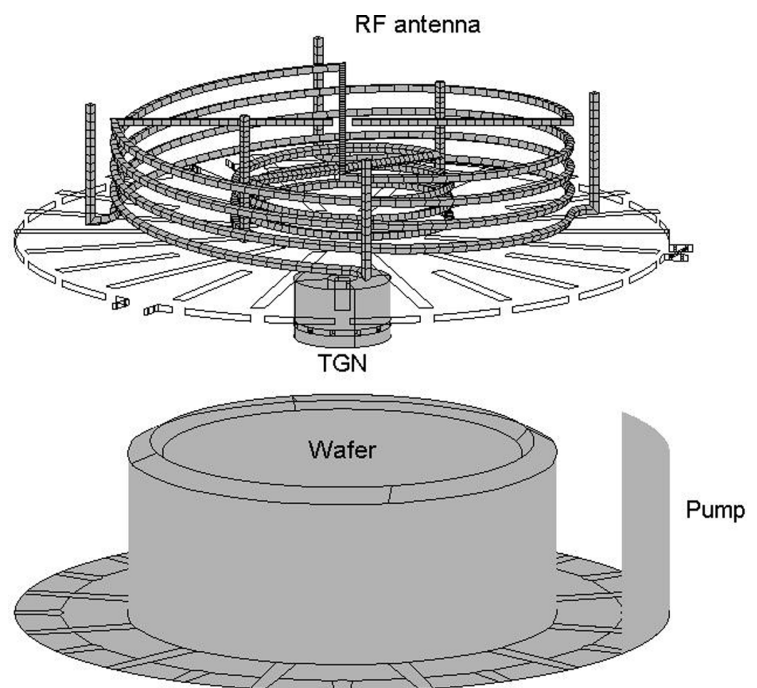

Figure 1. Geometrical configuration of the model.

research. RF antenna is a very tricky object in reducing necessary cells in modeling. There are two methods: a filament model and a solid model. Solid antenna models are consuming much more number of cells in constructing rf sections. A filament model has strong advantage in reducing necessary number of cells. But it has a weakness in junctions. The outer turn of DPS has a quadrature junction at the center region. A single rf feeding line is divided into four equal length of antenna sections. A model starting from the rf feeding point from the matching box would consume much longer CPU time in calculating converged electric potential profile, especially around the junctions. So we used a simplification scheme by placing 
the starting points of outer turn overlapped, but not connected. By using this method, the electric potential profile and the resultant current were calculated only after 6-7 iterations.

Gas injections are divided in two directions: horizontal and vertical. The distance between the gas nozzle and the wafer is around $150 \mathrm{~mm}$. Usual nozzle size is around $1 \mathrm{~mm}$ in diameter. With very low viscosity of the processing gas and operating conditions, the injected gas velocity profile can be kept until the wall of the chamber. Usual processing pressures are around 1 2 Pa. Vertical injection gas profile can be adjusted by drilling the holes slanted from the $\mathrm{z}$ axis. Poly-Si etching is affected more by radicals and ions are playing more important role in metal etching steps. The $\mathrm{z}$ position of horizontal gas injection holes is different in two etching recipes.

\section{Results and Discussion}

\section{Effects of gas dynamics}

The tunable gas nozzle has two inlet arrays: horizontal and vertical. When the vertical inlet holes are modeled 45 degree slanted, two vortexes were formed around the injection line. Twelve horizontal and vertical inlet holes are positioned alternatively by 15 degrees. TGN height is $50 \mathrm{~mm}$. The process gap is $150 \mathrm{~mm}$. The gas flow velocity magnitude from horizontal and vertical inlet are shown in Fig. 2 and Fig. 3. In vector representation in Fig. 4, the center axis region is showing up rising flow pattern from the wafer surface. And a vertical counter clockwise vortex is developed at the corner of the TGN and the ceramic lid. From this result, the etch byproduct from the center of the wafer would slowly fly off to the dead center region of the TGN and be mixed with newly injected fresh gases. For poly $\mathrm{Si}$ etching with $\mathrm{F}$ containing gases, byproducts is $\mathrm{SiF}_{4}$ and it would be bombarded in the donut shape region between the inner and outer rf antenna turn and then be dissociated or ionized to produce sub species: $\mathrm{SiF}_{3}{ }^{+}, \mathrm{SiF}_{3}$, etc. In CCP reactors, gases are injected from the top electrode's nozzles. Gases would make a parallel vertical flow from the top to bottom, making a simple flow pattern. In ICP reactors, the ceramic lid is necessary to make a rf transparent vacuum tight seal. Gas injection from the peripheral positions around the chamber would not penetrate deep into the reaction volume different from common experiences at atmospheric pressure. Usual processing gas pressure is around $2 \mathrm{~Pa}$.

The simulation result is showing upward flow toward the just below region between the inner and the outer $\mathrm{rf}$ antenna turns in Fig. 4. Processing gases are heavily bombarded by electrons at this region where the density of electrons has a peak. Dry etching process is a balance between polymer deposition and ion induced desorption of reacted surface mixture. Thermal desorption is a strong function of the surface temperature. Desorbed species can
DPSII lid1 heater1 TGN2 coilbox1a 10 mTorr H500 V500 45deg 1T vis5E7

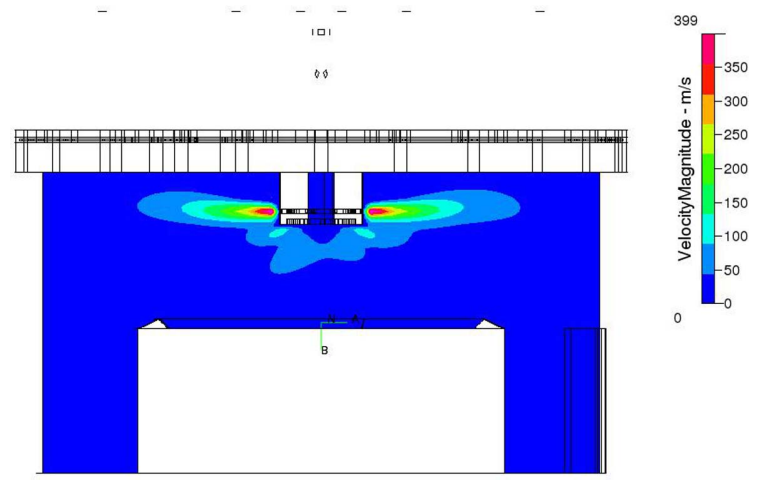

Figure 2. Gas flow velocity profile at the cutting plane of horizontal gas inlet nozzles.

\section{DPSII lid1 heater1 TGN2 coilbox1a 10 mTorr H500 V500 45deg 1T vis5E7}

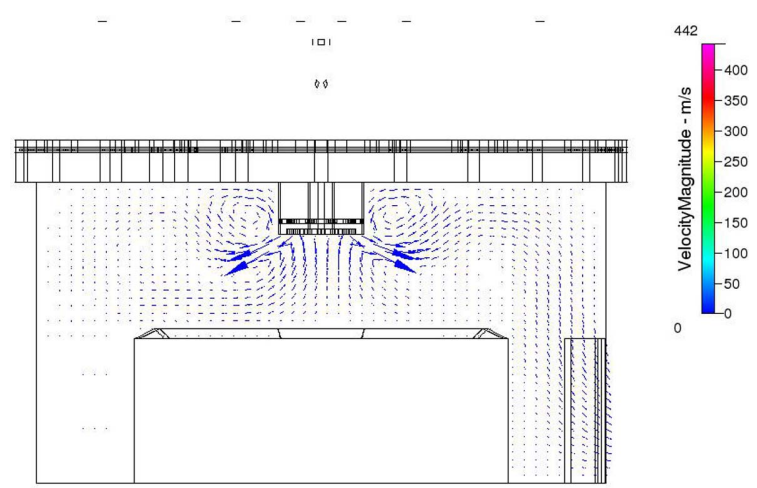

Figure 3. Gas flow velocity profile at the cutting plane of vertical gas inlet nozzles.

DPSII lid1 heater1 TGN2 coilbox1a 10 mTorr H500 V500 45deg 1T vis5E7

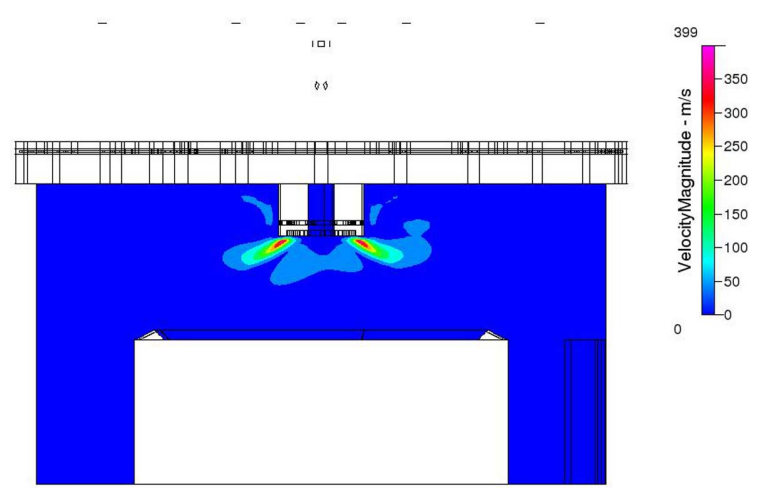

Figure 4. Gas flow velocity vector profile at the cutting plane of vertical gas inlet nozzles showing strong vortex.

be carried by forced convection as well as free diffusion.

In Fig. 5, massless particle traces showed gas flow from horizontal inlet nozzles. Flow velocity is reduced from $400 \mathrm{~m} / \mathrm{s}$ to slower than $50 \mathrm{~m} / \mathrm{s}$ within a few $\mathrm{cm}$ from the nozzle outlet faces. The kinematic viscosity was assumed to $5 . \mathrm{E}-7$ which is reasonable value at this pressure. The 


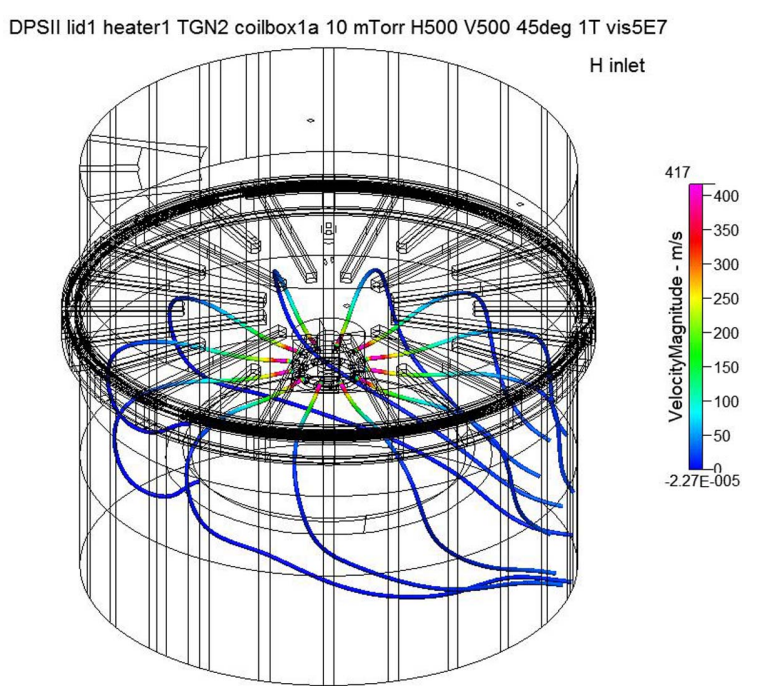

Figure 5. Massless particle traces from the horizontal gas inlet nozzles (perspective view).

DPSII lid1 heater1 TGN2 coilbox1a 10 mTorr H500 V500 45deg 1T vis5E7 $\mathrm{H}$ inlet
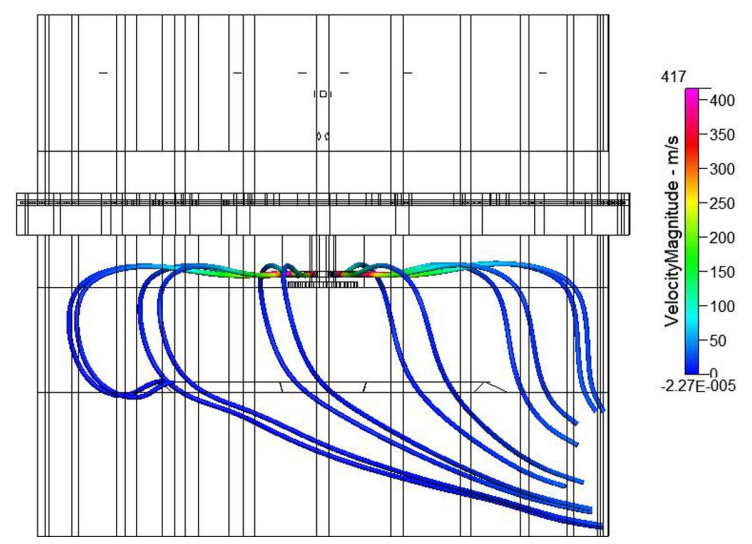

Figure 6. Massless particle traces from the horizontal gas inlet nozzles (side view).

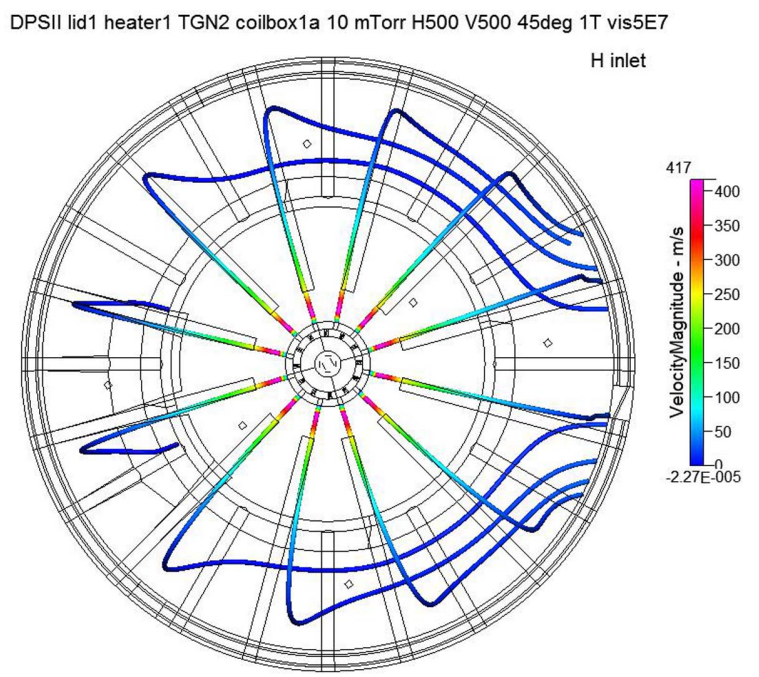

Figure 7. Massless particle traces from the horizontal gas inlet nozzles (top view).

side view of Fig. 5 is Fig. 6. Interesting point is the right half shows that gases injected from the horizontal nozzles
DPSII lid1 heater1 TGN2 coilbox1a 10 mTorr H500 V500 45deg 1T vis5E7

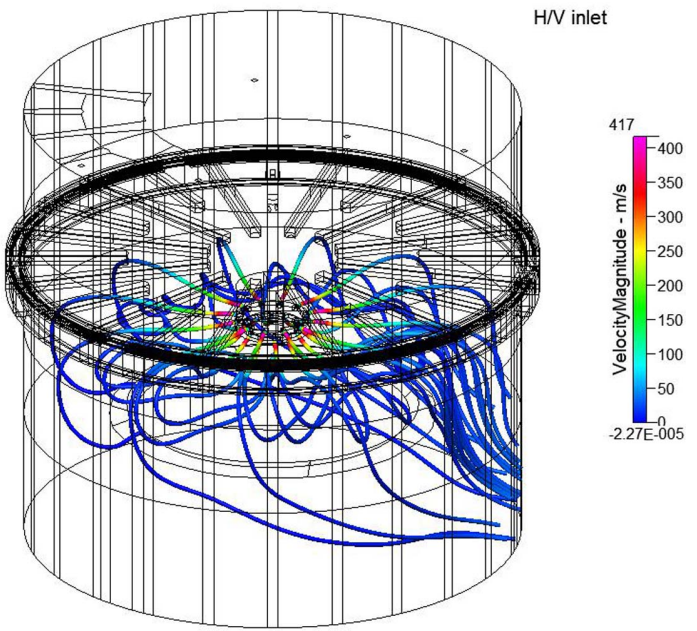

Figure 8. Combined view of massless particle traces from the horizontal and vertical gas inlet nozzles.

DPSII lid1 heater1 TGN2 coilbox1a 10 mTorr H500 V500 45deg 1T vis5E7

$\checkmark$ inlet

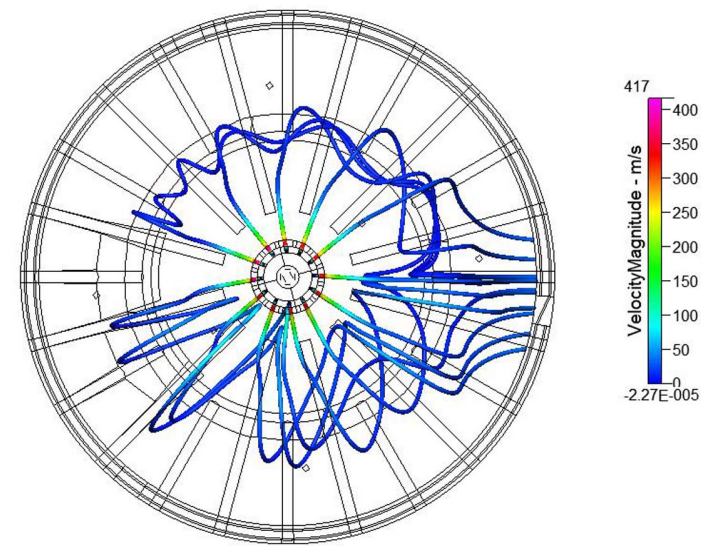

Figure 9. Massless particle traces from the vertical gas inlet nozzles (top view).

flow directly to the pump connection faces without hitting the wafer areas. The top view (Fig. 7) shows injected gases make turning around the chuck side walls. Gases from horizontal and vertical nozzles show added pattern from each cases. Vertical inlet nozzles are making collision against gas flow from the horizontal inlet nozzles at the edge of the wafer. As in Fig. 4, gases from the vertical nozzles hit the wafer area and recoil up to the lid. On the average, the residence time is calculated by the chamber volume/effective pumping speed. The computational flow dynamics reveals potential stagnant regions which cannot be pinpointed by common sense.

In Fig. 10, backward traces of massless particles are shown from the wafer surface. By using this, the origin of the incoming flow toward the wafer is the nozzles opposite to the pump. From this point of view, almost half of the injected processing gases are wasted directly to the pumping stack. The only way to use the right half of the gases is thermal diffusion which depends on the concentration gradient. Top view of this is shown in Fig. 
DPSII lid1 heater1 TGN2 coilbox1a 10 mTorr H500 V500 45deg 1T vis5E7

Wafer
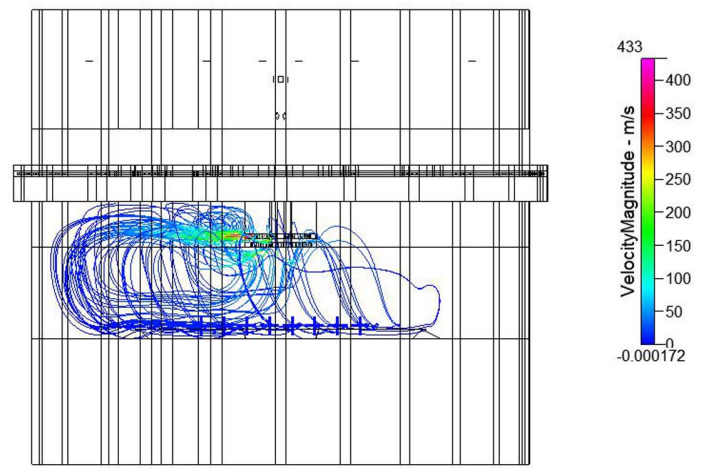

Figure 10. Backward traces of the gas flow incoming to the wafer (side view).

DPSII lid1 heater1 TGN2 coilbox1a 10 mTorr H500 V500 45deg 1T vis5E7

Wafer
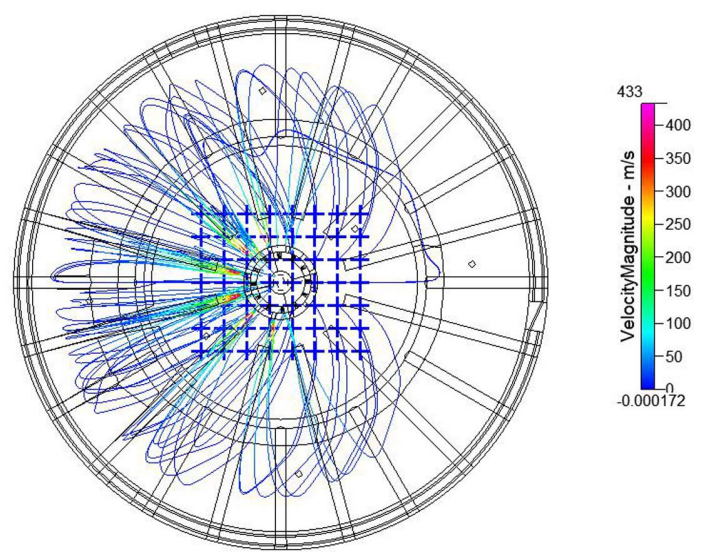

Figure 11. Backward traces of the gas flow incoming to the wafer (top view).

DPSII lid1 heater1 TGN2 coilbox1a 13MHz cond5E3 100V -50V Ar10 mTorr H500 V500 1T vis5E7

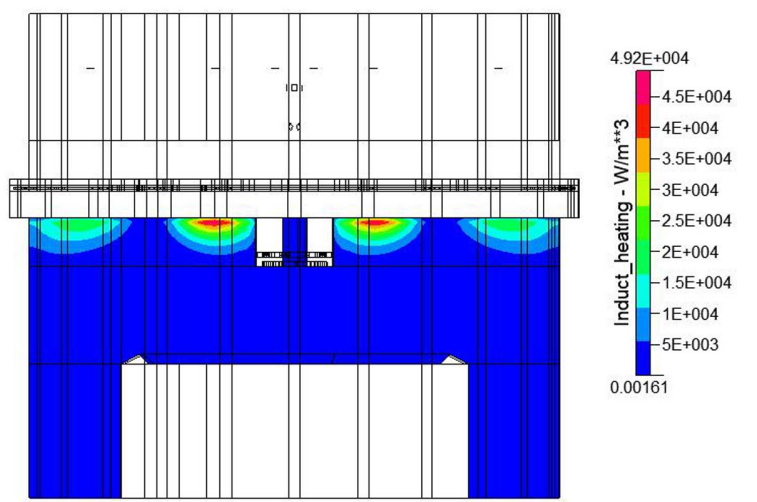

Figure 12. RF power absorption profile of the reverse current configuration.

11. It clearly shows evidence on the mention above.

\section{Effects of rf driving current direction}

A base model is developed with a condition of the same direction in rf driving currents. Usually, this would invoke
DPSII lid1 heater1 TGN2 coilbox1a 13MHz cond5E3 100V -50V

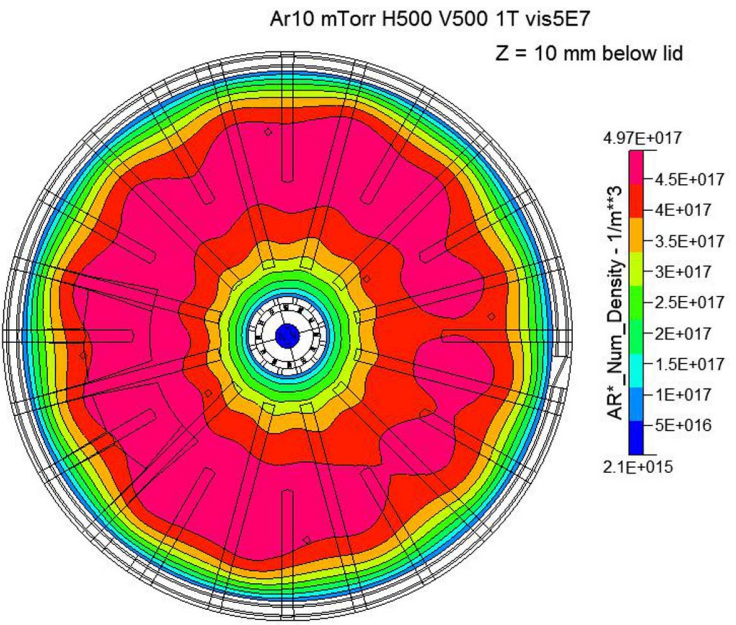

Figure 13. Side view of the vertical cut in rf power absorption profile.

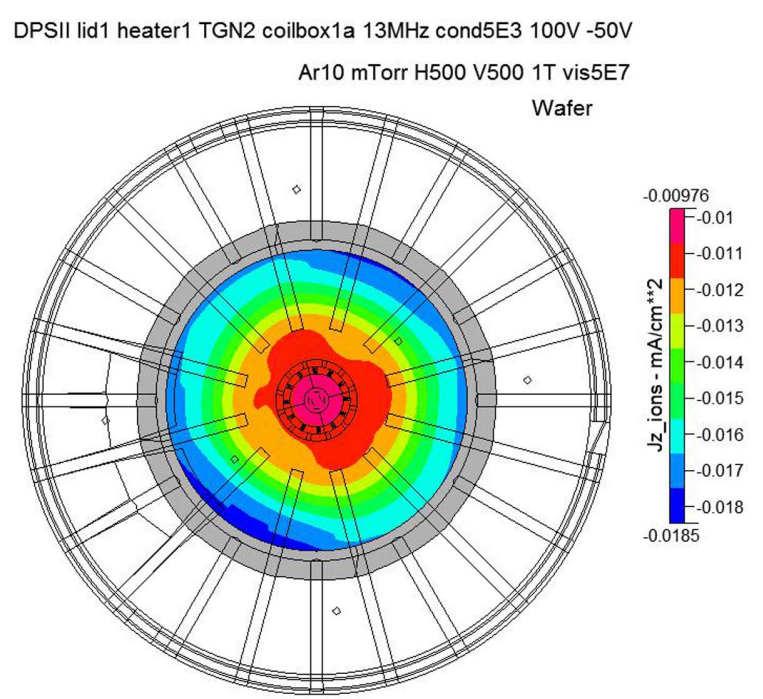

Figure 14. Top view of the z-cut in Ar metastable's number density profile.

rf power deposition region between the inner and the outer turns. In Fig. 11, the outer turn section of the rf antenna was set to have the opposite direction against the inner section by changing antenna end voltage. The rf power absorption region is separated to the core and the peripheral region. With parallel driving current direction configuration, the donut shape region between the inner and the outer turn of the rf antenna is the main area of power deposition. The side view of this is in Fig. 13. RF current of the inner turn was $14.9 \mathrm{~A}$ and that of the outer turn was $5.2 \mathrm{~A}$. While the rf power deposition pattern is opposite, the electron density profile is not affected much by this, because the wall recombination is the main factor at this operating gas pressure, $\sim 3 \mathrm{~Pa}$. A very interesting point in this result is neutral particle (the metastable state of Ar) profile at the z-cut of the horizontal gas inlet level shown in Fig. 14. With drift-diffusion approximation used in this study, charged particles would follow the electric field distribution and neutral particle's profile would be 


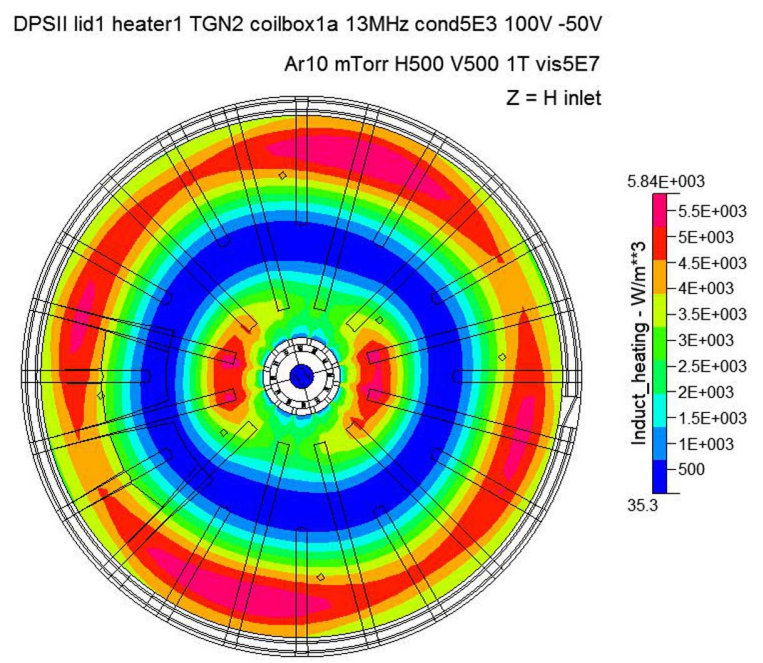

Figure 15. Vertically incident ion flux distribution onto the wafer.

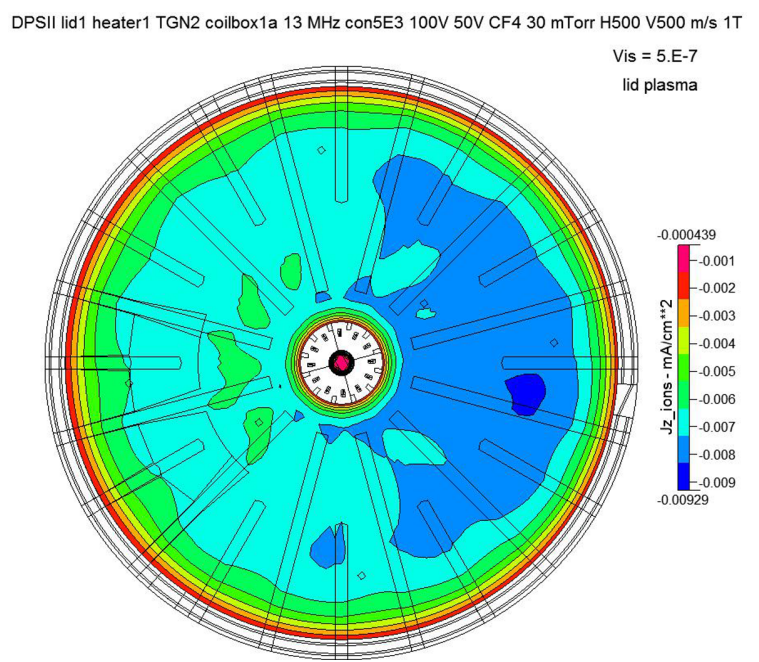

Figure 16. Gas flow velocity profile at the horizontal inlet level.

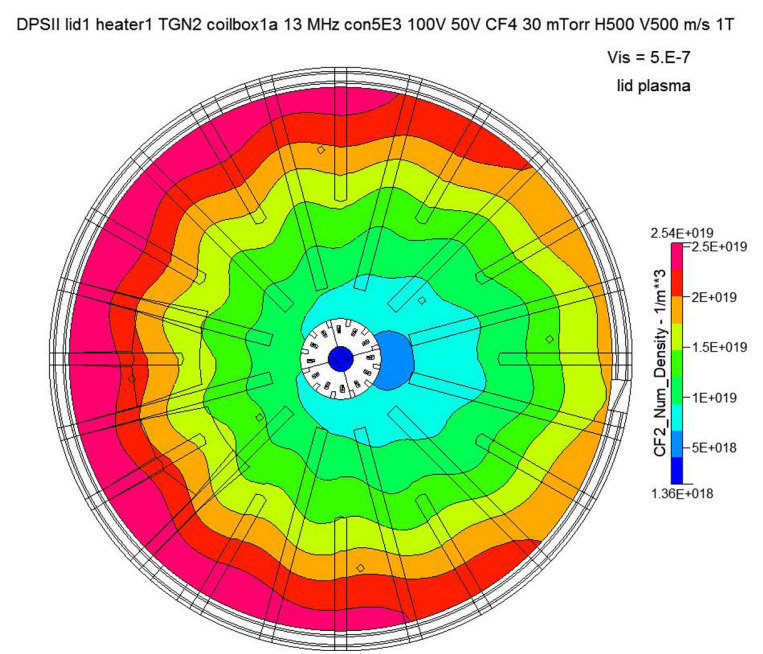

Figure 17. Vertically incident ion flux to the ceramic lid.

affected firstly by the gas flow dynamics, secondly by the multi-step collision processes. Ar metastables are generated by direct electron impact collisions. One advantage of the reverse current driving scheme would be correcting

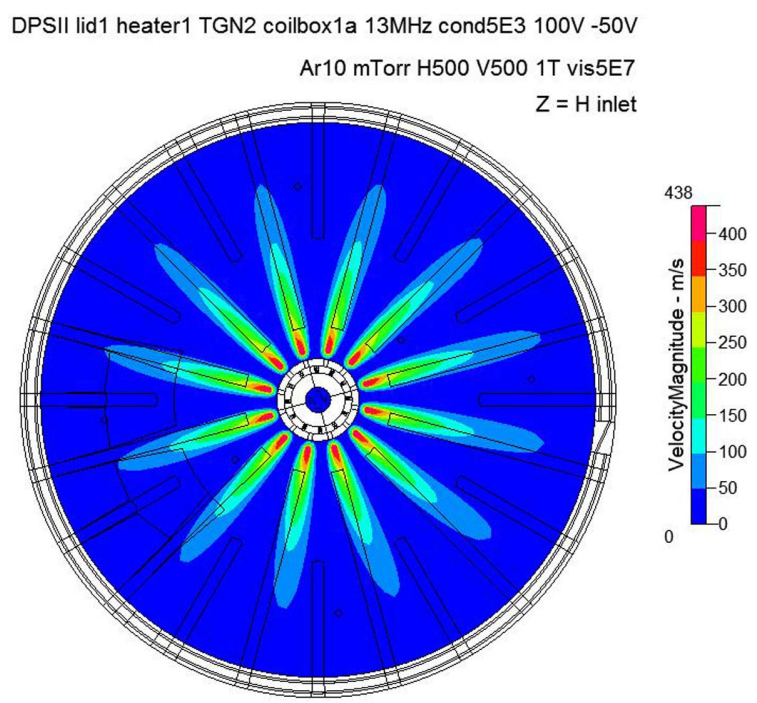

Figure 18. CF2 number density at the surface region of the ceramic lid.

excessive processing rate at the wafer center region. In Fig. 15 , the incoming flux of ions to the wafer is shown. The vertical component of ion flux at the center is $0.01 \mathrm{~mA} / \mathrm{cm}^{2}$ and that at the edge is $0.018 \mathrm{~mA} / \mathrm{cm}^{2}$. By adjusting the rf current of the outer turn, the ion flux ratio of the center and the edge region would be changed to enhance the processing uniformity. One disadvantage of the current setup would be the skewed profile by the inner turn antenna which is made of two twisted half turns and this would invoke two isolated profiles of rf power deposition in Fig. 12. Etching gases like $\mathrm{CF}_{4}$ would produce many radical species and etch byproducts. Gas flow dynamics would affect mainly the injected species, $\mathrm{CF}_{4}$ rather than produced ones by electron impact collisions. The high injected gas velocity profile would look like in Fig. 16. The Mach number at the nozzle face was calculated about 3.0. The lower surface of the ceramic lid would be bombarded by ions as shown in Fig. 17 and the ion flux is determined by the volume density of ions and the electric potential difference around the surface to draw ions from the bulk plasma through the developed sheath. After extended operation of the etch process, exposed surfaces would develop thick polymer layers composed of $\mathrm{C}$ and $\mathrm{F}$. From the simulation of $\mathrm{CF}_{4}$ plasma with the outer $\mathrm{rf}$ coil turned on, skewed $\mathrm{CF}_{2}$ radical profile was obtained as shown in Fig. 18. The right side is the pumping duct direction. Finally remaining polymer will be determined by radical's deposition and incoming ion's sputtering off.

\section{Conclusions}

The effects of gas inlet conditions and rf driving current on the plasma characteristics in a dual turn ICP reactor with a tunable gas nozzle. At a gas pressure of $2.25 \mathrm{~Pa}$, the horizontal and vertical gas inlet nozzles have affected the flow dynamics. With 45 degree inclined injection from the 
vertical inlets, distinct vortices were developed around the corner of the TGN and the center region between the TGN and the wafer. Numerical simulation showed the injected gases collide against the one from wafer region after touching the inner surface of the edge ring and go beneath the highest $\mathrm{rf}$ power deposition region with a parallel $\mathrm{rf}$ driving current scheme. In the reverse rf driving current configuration, the highest power absorption position was separated into two regions:two inside islands and four peaked donut along the outside of the outer rf turn. A simple CF4 chemistry simulation showed a skewed profile of the incoming ion flux and CF2 number density nearby the ceramic lid separating the rf antenna from the vacuum chamber. This will provide a clue in explanation of the polymer deposition profile on the lower surface of the ceramic lid after extended operation.

\section{Acknowledgements}

This research is supported by WC300 research project under contract no 10046957 by the Korean Ministry of Trade, Industry and Energy.

\section{References}

[1] D. Seo and S. Chun, J. Kor. Inst. Surf. Eng., Vol. 45, No. 3, 121 (2012).

[2] S. Lee, J. Kor. Vac. Soc., Vol 18, No. 3, 176 (2009).

[3] K. Yang, S. Park, T. Shin, and G. Yeom, J. Kor. Inst. Surf. Eng., Vol. 48, No. 6, 360 (2015).

[4] H. Park, J. Choi, M. Yun, and G. Kwon, J. Kor. Inst. Surf. Eng., Vol. 49, No. 1, 92 (2016).

[5] B. Lee and G. Jeong, J. Kor. Inst. Surf. Eng., Vol. 48, No. 4, 163 (2015).

[6] J. Joo, J. Kor. Inst. Surf. Eng., Vol. 46, No. 3, 133 (2013).

[7] S. Chun, J. Kor. Inst. Surf. Eng., Vol. 48, No. 5, 205 (2015).

[8] S. Chun and S. Lee, J. Kor. Inst. Surf. Eng., Vol. 49, No. 4, 376 (2016).

[9] S. Jung, W. Ahn, and D. Kim, J. Kor. Inst. Surf. Eng., Vol. 49, No. 1,87 (2016).

[10] J. Lim, S. Kim, D. Kim, M. Jeong, J. Lee, and W. Yun, Appl. Sci. Conv. Technol., Vol.24, No.5, 184 (2015).

[11] D. Kwon and N. Yoon, Appl. Sci. Conv. Technol., Vol 24., No. 6, 237 (2015). 